\title{
Nordiques
}

40 | 2021

Territoires de la migration dans les pays nordiques et baltes

Aspects des mouvements migratoires engendrés par la contribution industrielle britannique dans l'espace nordique durant la deuxième moitié du $\mathrm{XIX}^{\mathrm{e}}$ siècle

Philippe Blanc

\section{QpenEdition Journals}

Édition électronique

URL : https://journals.openedition.org/nordiques/1325

DOI : $10.4000 /$ nordiques. 1325

ISSN : 2777-8479

Éditeur :

Association Norden, Bibliothèque de Caen la mer

\section{Référence électronique}

Philippe Blanc, « Aspects des mouvements migratoires engendrés par la contribution industrielle britannique dans l'espace nordique durant la deuxième moitié du XIXe siècle », Nordiques [En ligne], 40 | 2021, mis en ligne le 02 mai 2021, consulté le 11 juillet 2021. URL : http://journals.openedition.org/ nordiques/1325; DOI : https://doi.org/10.4000/nordiques.1325

Ce document a été généré automatiquement le 11 juillet 2021.

Nordiques 


\title{
Aspects des mouvements migratoires engendrés par la contribution industrielle britannique dans l'espace nordique durant la deuxième moitié du $\mathrm{XIX}^{\mathrm{e}}$ siècle
}

\author{
Philippe Blanc
}

\section{Introduction}

Toutes les régions situées autour de la mer Baltique ont connu, depuis les $\mathrm{XVI}^{\mathrm{e}}$ et $\mathrm{XVII}{ }^{\mathrm{e}}$ siècles, plusieurs vagues migratoires venues des îles britanniques résultant soit des conflits qui ont bouleversé la région, amenant avec eux des milliers de mercenaires écossais, soit sous la forme de communautés marchandes qui se sont créées ici et là, de manière plus ou moins pérenne, comme autant de points stratégiques du commerce baltique de la Grande-Bretagne, essentiellement lié à l'importation de matières premières. Mais pour ce qui nous intéresse dans le présent article, la structuration de ces communautés après les années 1850 ne ressemble en rien à celles des XVII ${ }^{e}$ et $\mathrm{XVIII}^{\mathrm{e}}$ siècles, composées alors de petits marchands, de boutiquiers et d'artisans. En effet, en dehors de l'activité commerciale, certes primordiale, le bouleversement qui modifie la nature de l'influence britannique au Nord de l'Europe à ce moment-là est tout naturellement causé par l'industrialisation. Celle-ci affecte petit à petit le continent et ne manque pas de laisser une empreinte très visible sur le plan démographique, même s'il faut garder à l'esprit, à travers toute cette étude, que la présence britannique dans la région, bien que venant en deuxième position sur le plan quantitatif, est sans commune mesure avec la présence allemande. Les chiffres cités pour Saint-Pétersbourg, par exemple, sont sans équivoque à ce sujet, avec trois mille 
résidents britanniques pour quatre-vingt mille Allemands (en 1871). Les rapports sont les mêmes sur les deux autres centres les plus importants du pourtour baltique : quatre cents Britanniques pour mille huit cents Allemands à Christiania ${ }^{1}(1871)$, et cent cinquante Britanniques pour cinq mille cinq cents Allemands à Riga (1867). Seule Göteborg possède sensiblement le même nombre de résidents britanniques et allemands (cent cinquante en 1871) ${ }^{2}$. En ce sens, nous devons davantage parler de colonies que de migration de masse, mais des colonies suffisamment implantées et actives socialement pour posséder au moins un véritable lieu de culte officiel, anglican notamment ${ }^{3}$.

\section{La nature de l'apport industriel britannique dans le Nord}

\section{Les « entrepreneurs »}

2 Depuis la fin du XVIII siècle, l'Europe du Nord a vu arriver un certain nombre d'immigrés « entrepreneurs ", à la fois « capitalistes, innovateurs, patrons, marchands et vendeurs $»^{4}$, créant des usines dans un cadre très restrictif quant à l'exportation de technologie et de savoir-faire, surtout jusqu'en 1825. Ces grands noms sont très connus : ils vont de l'archétype dans la région, William Cockerill $(1759-1832)^{5}$, à Charles Gascoigne, patron des aciéries Carron de Falkirk en Écosse ${ }^{6}$, en passant par l'autre Écossais emblématique, Charles Baird (1766-1843), spécialisé dans l'industrie mécanique à Saint-Pétersbourg7 . Le Grand-Duché de Finlande voit, quant à lui, arriver en 1823 James Finlayson (1772( ?)-1852), né à Glasgow, à l'origine des filatures de coton qui feront la célébrité de Tammerfors (Tampere). En Suède ${ }^{8}$, où la région de Göteborg, véritable tête de pont écossaise depuis le $\mathrm{XVII}^{\mathrm{e}}$ siècle ${ }^{9}$, occupe une place prépondérante, nous mentionnons en particulier les deux associés William Gibson (1783-1857) et Alexander Keiller (industrie textile et chantiers navals) ${ }^{10}$, mais aussi Samuel Owen (1774-1854), qui monte en 1809 son propre atelier mécanique, le Kungsholmens Mekaniska Verkstad, à Stockholm ${ }^{11}$, et, à partir des années 1830, les frères Alexander et Andrew Malcolm, spécialisés dans le secteur textile à Norrköping ${ }^{12}$.

Toutefois, l'apport britannique à l'effort industriel et à la modernisation nordiques va prendre une tout autre dimension à partir du milieu du XIX ${ }^{e}$ siècle, d'une part grâce à la libéralisation totale par la Grande-Bretagne de l'exportation de technologie, de savoirfaire et de personnels qualifiés $(1843)^{13}$, et d'autre part parce que cette contribution bénéficie d'une phase de transformation et d'expansion socioéconomique qui s'engage dans le Nord du continent européen ${ }^{14}$.

\section{La participation industrielle britannique change de nature}

Si la modernisation à géométrie variable du Nord de l'Europe intéresse en premier lieu une partie des détenteurs de capitaux britanniques qui n'hésitent pas à investir à l'étranger, nous nous pencherons davantage ici sur les hommes qui transforment un pari financier en des avancées concrètes.

5 La guerre de Crimée à peine terminée, la revue professionnelle The Engineer exprimait l'année même de sa fondation (1856), dans une tirade digne de l' «impérialisme du libre-échange $»^{15}$, des ambitions clairement affichées : 
Qui ne peut prévoir dans ces judicieux projets [de construction ferroviaire ${ }^{16}$ ] le développement de la véritable force de l'Empire russe, et une extraordinaire occasion des plus rémunératrices pour les compétences pratiques et le talent anglais ? ${ }^{17}$

6 Cinq ans après l'exposition universelle de 1851, voilà donc le petit monde de l'encadrement industriel britannique, apparemment tout imprégné de ce sentiment de supériorité confiant et non dénué d'un certain esprit de conquête, prêt à partir au nom de cette forme spécifiquement matérialiste d'œuvre civilisatrice, dans laquelle les techniciens et les ingénieurs britanniques ne sont plus des entrepreneurs individuels, mais deviennent une armée de missionnaires. C'est ainsi que, quelles que soient les motivations personnelles de tous ces hommes,

les continents d'Europe et d'Amérique [...] fourmillent d'Anglais et d'Écossais qui, conservant toutes leurs caractéristiques nationales, emportent avec eux dans tant de pays étrangers ces avancées scientifiques et artistiques qui civilisent et font progresser d'autres nations moins privilégiées que la nôtre. ${ }^{18}$

7 Mais quelle est la réalité de cette «œuvre civilisatrice »? Même en l'absence de statistiques précises, nous pouvons estimer avec R.A. Buchanan que grâce à «la prédominance quasiment incontestée de la Grande-Bretagne dans le domaine de l'expertise mondiale d'ingénierie durant la deuxième moitié du XIX ${ }^{\mathrm{e}}$ siècle, [...] la diaspora [de l'ingénierie britannique à l'étranger] a atteint son apogée entre 1850 et $1914 »^{19}$. Or, nous allons le voir, cela est précisément le cas durant cette période autour de la Baltique.

\section{Vision d'ensemble des évolutions nordiques}

8 Il est illusoire de vouloir effectuer un recensement précis des effectifs industriels britanniques dans l'espace nordique pour la période qui nous intéresse; les sources ne sont que trop rares ou du moins parcellaires. Il est en revanche possible de se faire une idée relative de la répartition de ces effectifs. Il existe en effet certaines sources mentionnant la présence d'ingénieurs ou de techniciens à l'étranger, parfois à un moment précis. Pour notre part, nous avons pu établir des représentations sous forme d'histogrammes (Figures 1 et 2) grâce aux renseignements fournis par quelques auteurs, mais surtout grâce aux archives londoniennes des deux principales associations d'ingénieurs britanniques, à savoir The Institution of Civil Engineers et The Institution of Mechanical Engineers. Ont été également d'un grand secours les Archives nationales britanniques (The National Archives) et les archives municipales de Londres (London Municipal Archives) qui détiennent les registres de naissances, de mariages et de décès de certaines communautés britanniques vivant à l'étranger. Ces sources ont bien sûr leurs limites puisqu'elles ne conservent aucune trace des « électrons libres", en particulier les célibataires sans enfants n'ayant appartenu à aucune association. Les nombres donnés par les histogrammes, à savoir deux cent quatre-vingt-dix personnes environ, ne représentent ainsi que le « noyau » des effectifs industriels, qui sont donc bien supérieurs en réalité. Toutefois, l'intérêt des Figures 1 et 2 est surtout de nous donner à la fois un aperçu des différentes catégories socioprofessionnelles (par employeurs) présentes des deux côtés de la Baltique et de nous indiquer leur évolution entre les années 1850 et la Première Guerre mondiale. 
Figure : Évolution de la présence attestée des effectifs industriels britanniques, principalement d'encadrement, en Scandinavie entre 1850 et $1915^{20}$

\section{Scandinavie}

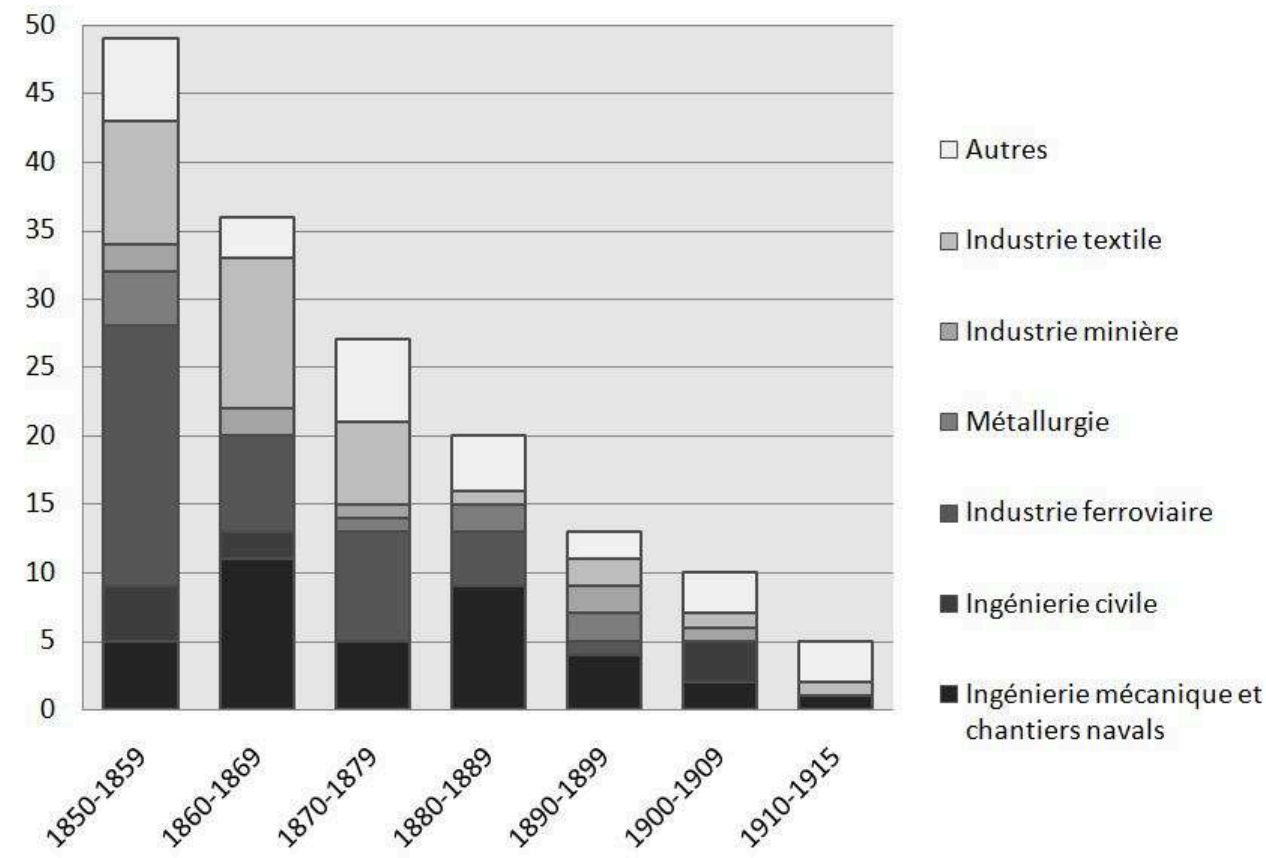

Figure . Évolution de la présence attestée des effectifs industriels britanniques, principalement d'encadrement, en Russie baltique entre 1850 et $1915^{21}$

\section{Russie baltique}

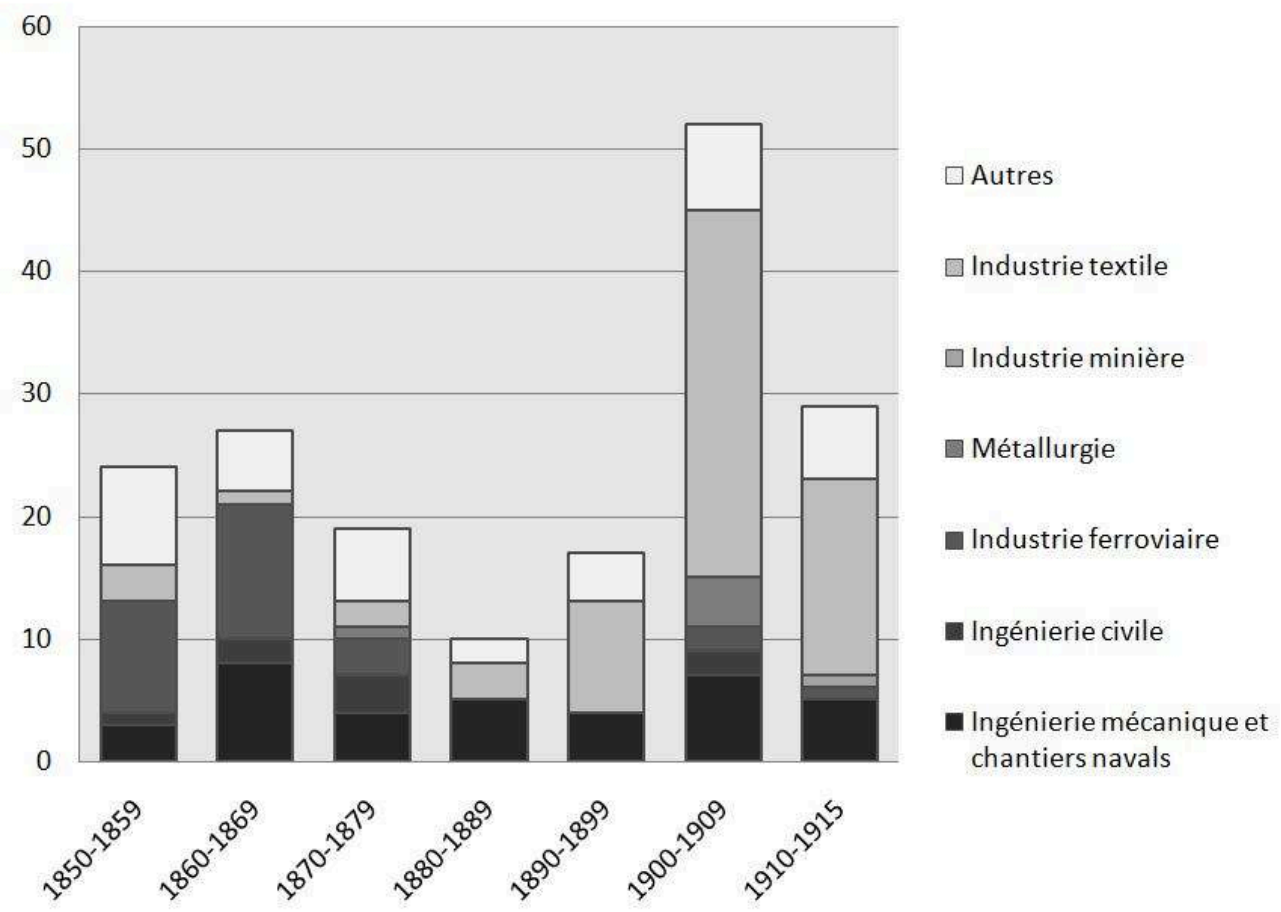




\section{Les évolutions générales en Scandinavie}

Concernant la Scandinavie, tout d'abord, l'évolution de la Figure 1 est frappante, même si sa régularité est en trompe-l'œil. Comme le fait remarquer le consul britannique à Christiania (Oslo) J.R. Crowe en 1848 : « La majeure partie des résidents britanniques en Norvège [est] constituée d'ingénieurs, de machinistes et d'ouvriers, $»^{22}$ et cette présence peut encore être considérée comme importante passé le milieu du XIX ${ }^{e}$ siècle, tandis qu'elle semble décroître de manière inexorable, surtout au cours des années 1880 , avec trois domaines de prédilection : l'industrie mécanique, l'industrie textile et les chemins de fer, dont nous aurons à reparler.

C'est que la présence industrielle britannique en Scandinavie obéit à un schéma bien particulier, à savoir qu'encore au milieu du XIX ${ }^{\mathrm{e}}$ siècle la Suède, par exemple, tout comme l'industrie textile norvégienne, se trouvent «avoir besoin des connaissances anglaises et écossaises ${ }^{23}$ dans le cadre du transfert de technologie nécessaire qui s'opère et augmente au moins depuis $1825^{24}$. Mais sur place, contrairement à l'époque où les entrepreneurs anglais ou écossais s'installaient souvent définitivement, le rôle de la foule de techniciens, ouvriers qualifiés et ingénieurs britanniques est devenu beaucoup plus provisoire; il s'agit surtout à présent de s'approprier certains savoirfaire, condition sine qua non d'une maturité ouvertement recherchée, à mesure que s'industrialise le pays. C'est en partie ce à quoi sert l'usine Motala Verkstad, créée en 1822 par le comte Baltzar von Platen et conçue comme devant devenir le fournisseur principal en machines de tout le pays. L'usine devient en effet un véritable centre de formation britannique " pour ingénieurs et techniciens ${ }^{25}$ suédois puisque «l'expertise technique à Motala est représentée à l'époque entièrement par des Anglo-Saxons » avec pendant longtemps à leur tête un directeur écossais, Daniel Fraser, que l'on retrouvera entre 1858 et 1862 construisant la plus grande fabrique de papier d'Europe à SaintPétersbourg ${ }^{26}$.

11 Ce rôle d'instructeur-formateur-superviseur se généralise assez vite en Suède et se rencontre bien au-delà de Motala, y compris dans des secteurs clés suédois comme la métallurgie, avec des techniciens britanniques très vite relayés par leurs collègues suédois ${ }^{27}$, tandis que l'on assiste à un phénomène un peu similaire du côté norvégien, en particulier dans l'industrie textile ${ }^{28}$. En l'occurrence les années 1870-1880 constituent un tournant ${ }^{29}$, durant lequel on assiste à une diminution sensible des effectifs industriels britanniques, comme le confirme l'examen de la Figure 1.

\section{Un contexte russe très différent}

12 Malgré quelques points communs, notamment l'importance des secteurs ferroviaire et mécanique dans les années 1850-1860, la lecture de la Figure 2 offre un contraste saisissant avec la Figure 1 en ce sens que l'on voit les effectifs britanniques non pas diminuer, mais exploser durant les deux décennies qui précèdent la Première Guerre mondiale alors même que l'industrialisation russe est, elle aussi, en pleine expansion, surtout après 1890. On peut donc légitimement se demander d'où provient une telle différence.

Prenons l'exemple de l'industrie textile qui, outre son rôle de vecteur de base de l'industrialisation, constitue également le principal domaine d'intervention de la maind'œuvre britannique. La région baltique devient assez vite l'une des trois grandes 
régions textiles de Russie, et l'on ne peut manquer de parler de Ludwig Knoop (1821-1894), Brêmois mais Anglais d'adoption, venu en Russie dans les années 1840 depuis Manchester, où il était employé par la firme De Jersey ${ }^{30}$. Celui-ci fonde une usine à Narva, sur l'île de Kreenholm en $1857^{31}$, qui devient rapidement une véritable porte d'entrée dans l'importation et la diffusion de machines anglaises au sein de cette industrie ${ }^{32}$, essentiellement fournies par des firmes de la région de Manchester : Platts, Dobson \& Barlow, John Musgraves \& Sons, Hargreaves, Howard \& Bullough et Samuel Brooks ${ }^{33}$.

$14 \mathrm{Si}$, comme en Scandinavie, on fait appel à la main-d'œuvre britannique, au moins pour faire fonctionner les machines, ce phénomène ne fait ici que s'amplifier graduellement, et l'on assiste à une migration qui touche, pour ainsi dire, tous les échelons de l'entreprise, mais surtout les dirigeants et les cadres ${ }^{34}$. C'est le cas à Kreenholm, où, déjà en 1861, le directeur et le personnel de direction et de contrôle sont anglais (voir cidessous $)^{35}$. Mais au-delà de cet archétype, dans la capitale russe même, on peut mentionner parmi les employeurs de personnels britanniques bien d'autres usines dans le secteur du coton russe, à commencer par les filatures Ochta, fondées par les directeurs de la De Jersey \& Co., Johann Andreas et Johann H. Frerichs ${ }^{36}$. Dans cette même activité industrielle, la famille Hubbard lance en 1842 sa première compagnie textile en Russie: la Petroffsky Cotton Spinning and Weaving Manufacturing Company $^{37}$; mais la présence de personnels (et de matériels) britanniques ne se limite pas non plus au secteur du coton et déborde sur l'industrie lainière, à l'instar, dans les années 1850-1860, de la Egerton, Hubbard \& Co., et de la Thornton \& Co., créée en 1860 et elle aussi dirigée par un encadrement britannique ${ }^{38}$.

C'est pour cela que l'on peut dire que lorsqu'éclate la Première Guerre mondiale, «toutes les usines textiles de Saint-Pétersbourg [...] sont dirigées par du personnel technique britannique, en général un directeur, son assistant, un cardeur, un tisserand, et un ingénieur ${ }^{39}$. Cette situation se voit également dans d'autres centres industriels russes, notamment à Riga (voir ci-dessous), et c'est précisément ce qui explique le contraste observé dans les graphiques entre l'Ouest et l'Est de la Baltique. Si, grâce à un processus d'industrialisation déjà entamé et à des savoir-faire locaux traditionnels déjà bien ancrés ${ }^{40}$, la Scandinavie a su tirer le meilleur parti des connaissances et des formateurs britanniques pour s'en affranchir, en Russie, à l'environnement socioéconomique si différent, ces usines doivent de toute façon faire face à une pénurie de cadres locaux. Les spécialistes étrangers sont donc absolument nécessaires ${ }^{41}$, et ce de manière plus pérenne.

\section{Les domaines privilégiés de la contribution technologique britannique}

16 Il nous semble à ce stade essentiel de faire une présentation de quelques personnages emblématiques et de leurs champs d'intervention, dans la mesure où ce sont leurs initiatives industrielles qui donnent l'impulsion décisive aux mouvements migratoires de cadres et de main-d'œuvre dont nous parlons ici et dont ils font eux-mêmes partie dans certains cas. 


\section{La figure de proue : I'industrie ferroviaire}

17 Le symbole de la modernisation industrielle au milieu du XIX ${ }^{\mathrm{e}}$ siècle, et ceci dans le monde entier, reste le développement du chemin de fer. Et ce n'est pas un hasard si nos histogrammes montrent une présence significative de Britanniques dans ce secteur à ce moment-là. C'est en effet dans les années 1850 et 1860 - plus tardivement donc qu'en Europe occidentale - que se mettent en place les premières lignes publiques nordiques. Étant donné l'enjeu économique et technologique que celles-ci représentent, il n'est donc pas étonnant de voir se mettre sur les rangs la fine fleur de l'ingénierie britannique.

Le début des années 1850 - en 1851, 1852 et 1854 pour être précis ${ }^{42}$ - voit arriver en Norvège le grand Robert Stephenson (1803-1859), seul fils de George Stephenson, traditionnel "créateur du chemin de fer ", en vue d'effectuer les repérages pour la construction de la première ligne de chemin de fer du pays, construite entre Christiania et Eidsvoll, la Norsk Hoved-Jernbane ${ }^{43}$, avec à ses côtés George Parker Bidder (1806-1878). Très présent dans la Baltique, on retrouve ce dernier également, à peu près à la même époque, ingénieur en chef des chemins de fer danois (Royal Danish Railways) en partenariat avec la firme Peto \& Betts à partir de $1853^{44}$. Ce partenariat est assez caractéristique du microcosme de l'élite industrielle que l'on rencontre au cœur de ces premiers grands travaux ferroviaires danois et norvégiens, avec des noms parmi les plus célèbres au monde dans ce domaine, et travaillant souvent en association, à commencer par Samuel Morton Peto (1809-1889), qui a tout de même déjà à son actif la réalisation de grands travaux ferroviaires et monumentaux en Grande-Bretagne et ailleurs dans le monde ${ }^{45}$. Nous venons de le voir au Danemark, de 1848 à 1866, S. Morton Peto forme un partenariat avec son beau-frère, Edward Ladd Betts (1815-1872), employé de George Stephenson dans sa jeunesse ${ }^{46}$. Les combinaisons sont assez élastiques et répétitives puisque S. Morton Peto a en fait déjà travaillé avec G.P. Bidder sur la ligne norvégienne en 1851 et 1854 notamment ${ }^{47}$. Au Danemark, E.L. Betts et S. Morton Peto se retrouvent en outre associés à un autre personnage clé dans l'industrie ferroviaire, à savoir Thomas Brassey (1805-1870), lui aussi présent en Norvège ${ }^{48}$. Un autre ingénieur de même envergure est John Hawkshaw (1811-1891). Celui-ci est en effet à l'origine d'une quantité impressionnante de grands travaux d'ingénierie civile à travers le monde. On le retrouve dans la Baltique en Norvège, mais surtout en Russie dès après la guerre de Crimée, responsable de la construction de la ligne ferroviaire Riga-Dünaburg (Daugavspils) et assistant-ingénieur sur l'extension Dünaburg-Vitebsk de 1857 à 1861 et de 1862 à 1867 respectivement ${ }^{49}$.

\section{Le secteur de la construction mécanique}

Nous l'avons vu, l'industrie textile est souvent le point d'entrée des techniciens britanniques et en la matière, les flux connaissent un développement spectaculaire en Russie au tournant du siècle. Par ailleurs, Kristine Bruland a très bien montré la dimension du phénomène en Norvège. En Suède, il apparaît clairement que le secteur emploie encore des sujets de Sa Gracieuse Majesté au moins jusqu'à la fin des années 1870. Les Figures 1 et 2 pointent également l'importance persistante sur presque toute la deuxième moitié du XIXe siècle dans toute la zone baltique de la nécessité d'avoir du personnel britannique dans les domaines de la construction mécanique à la suite de ce qu'ont créé les Samuel Owens et les Charles Baird. En Suède, la famille Keiller, par 
exemple, est toujours présente aux chantiers navals d'Hisingen, près de Göteborg, jusqu'en $1906^{50}$, tandis que du côté russe, on trouve à Saint-Pétersbourg dans les années 1860 un partenariat d'ingénierie navale spécialisée dans la construction de navires de guerre composé de Charles Mitchell (1820-1895), Charles Swan (1831-1879) et Henry Swan (1842-1908) $)^{51}$. Dans le même domaine, un certain Murdoch MacPherson, présent en Russie dès 1839, fonde en 1856 les chantiers navals Baltic Shipyard, qui emploieront jusqu'à soixante-dix familles écossaises ${ }^{52}$. À Riga sont mentionnés dans les années 1860 les chantiers navals Lange \& Sons, ainsi que la fonderie Richard Poole ${ }^{53}$. Des Britanniques sont même employés en 1890-1891 pour superviser les travaux d'extension du port de Libau (Liepāja) ${ }^{54}$, destinée pourtant à devenir une importante base navale, et l'on voit des Écossais construire à Åbo (Turku) des navires à vapeur pour le gouvernement russe $(1866)^{55}$.

\section{Autre emblème de la participation britannique à la modernisation : les services urbains}

20 Les services urbains constituent eux aussi des secteurs de prédilection à mesure que les villes du Nord se modernisent. Le grand spécialiste de ce secteur est sans nul doute Henry A. Milne qui installe des réseaux de gaz à Malmö (1852-1854), Hambourg, Copenhague et dans de nombreuses villes suédoises (Landskrona et Gävle entre autres) ${ }^{56}$. C'est aussi un certain John Simpson que l'on trouve en 1876 dirigeant l'usine à gaz de Majarna à Göteborg ${ }^{57}$. Un cas un peu particulier est celui de Thomas A. English (1819-1889) : né à Copenhague, il est considéré comme le "pionnier de l'ingénierie civile au Danemark » où il travaille en partenariat avec C.J. Hanssen, Thomas Hawksley et G.P. Bidder à construire le réseau de gaz de sa ville natale (la Danish Gas Company). Il a également à son actif de nombreuses réalisations du même type, y compris dans la distribution d'eau et l'assainissement à Odense, Elseneur, Aalborg, Randers, Aarhus et Flensburg ${ }^{58}$. Quant à T. Hawksley (1807-1893) lui-même, il est ni plus ni moins le constructeur d'un réseau de distribution d'eau à Stockholm ${ }^{59}$, tandis que Francis Webb Wentworth-Sheilds (1820-1906) s'occupe du réseau d'assainissement de Riga ${ }^{60}$ et un autre spécialiste, John Francis Haseldine (1886-1944), fait la même chose à SaintPétersbourg (1908-1909) ${ }^{61}$.

\section{Caractéristiques de l'encadrement britannique}

\section{Les besoins spécifiques des réseaux ferrés...}

21 Si la direction suprême d'une partie des grands travaux ferroviaires nordiques est aux mains d'une poignée de compagnies londoniennes et de grandes célébrités de l'ingénierie britannique, ceux-ci ne peuvent mener à bien leur tâche que grâce à une armée de bras, bien sûr, mais aussi d'échelons intermédiaires de direction et de supervision locale travaillant sous leurs ordres, des compatriotes qui les assistent dans diverses tâches. Morgan Bransby Williams (1825-1914), par exemple, suit John Hawkshaw en Russie en tant qu'ingénieur en chef sur la construction de la ligne RigaDünaburg, puis en tant que responsable de son extension vers Vitebsk, dont il deviendra même le directeur pendant vingt ans ${ }^{62}$. Toujours sous les ordres de John Hawkshaw, on trouve aussi un « ingénieur-résident » sur la ligne Riga-Dünaburg en la 
personne de Thomas Colclough Watson ${ }^{63}$, qui avait déjà occupé cet emploi pour la Royal Swedish Railway à Örebro ${ }^{64}$.

Les charges de supervision des travaux et de direction des lignes, une fois celles-ci achevées, sont encore plus nombreuses en Scandinavie. Dès les premières interventions britanniques, nous trouvons déjà en 1852 John Rennie (1794-1874), ancien collaborateur de George Stephenson sur la mythique ligne Liverpool and Manchester Railway, nommé ingénieur en chef de la Kongliga svenska aktiebolaget för jernväg mellan Köping och Hult ${ }^{65}$. L'ingénieur William Jerry Walker Heath (1835-1869), lui, vient diriger la construction du chemin de fer près de Malmö en 1856-185766, et, à la fin de la décennie suivante, c'est un certain Walmsley Williams, que l'on voit en charge de la construction d'une ligne entre Frövi et Ludvika: la Swedish Central Railway, créée dès $1870^{67}$. Toujours en Suède, les années 1870 voient arriver un autre ingénieur anglais en vue de s'occuper de la construction de la ligne Halmstad-Nässjö: Alexander Woodlands Makinson (1822-1886) ${ }^{68}$, et c'est encore un autre Anglais, John Waddington Mann (1847-1894), que l'on nomme ingénieur mécanique en chef lors de la construction de la ligne " la plus septentrionale du monde ", la Swedish and Norwegian Railway, créée en 1883 et essentielle sur le plan économique puisqu'elle relie les gisements de fer de Gällivare et Kiruna au port de Luleå, sur le golfe de Botnie (1888), où l'ingénieur réside de 1886 à $1892^{69}$. Au Danemark, nous pouvons au moins mentionner Robert Charles Lindley (1824-1887), employé pendant trois ans en tant que "responsable de la construction de la plupart des premières lignes $»$ du pays ${ }^{70}$.

\section{... mais une omniprésence aux niveaux les plus élevés dans tous les domaines}

23 La complexité des réseaux ferrés est particulière, mais l'encadrement britannique s'observe tous azimuts. Nous en avons déjà évoqué les raisons, s'il est un domaine dans lequel celui-ci est jugé indispensable, c'est celui de l'industrie textile, et d'abord en Russie. Pour donner figure humaine à des raisonnements généraux, la Kreenholm, tout d'abord, se voit dirigée, par exemple, par un certain Richard Pilling en $1894^{71}$. Mais mentionnons aussi, entre autres, dans la capitale russe, John Williams, directeur des filatures de coton Ochka au moins entre 1905 et $1909^{72}$, John Albert Cheshire (1856-1907), propriétaire de la Cheshire Weaving Factory ${ }^{73}$, et dans le secteur lainier, John Thornton (1848-1908), propriétaire de la Thornton Woollen Mill ${ }^{74}$. Sinon, nous trouvons à Reval (Tallinn) un certain John Carr (1849-1911), directeur des filatures de coton $\mathrm{Baltic}^{75}$, et trois directeurs britanniques aux grandes filatures de coton de Strasdenhof, à Riga, entre 1875 et 1900 : Charles Hutton-Hall, William Sutcliffe, et V.E. Buckley ${ }^{76}$.

Le secteur mécanique n'est pas en reste, avec un certain Thomas Trenery, directeur d'une usine mécanique à Bergen à la fin des années $1860^{77}$, William George Riddell (1871-1954), ingénieur et directeur d'une usine de construction mécanique et de chantiers navals à Åbo au début des années $1900^{78}$. Comme nous l'avons déjà vu, les secteurs sensibles ne sont pas épargnés en Russie puisqu'un certain William Henry Greenwood (1846-1905) se voit propulser ingénieur en chef des aciéries et usines d'armement Abouchoff, à Saint-Pétersbourg (1875-1880) ${ }^{79}$.

Dans la partie occidentale de l'aire baltique, c'est surtout dans le domaine de l'exploitation des ressources naturelles au tournant du siècle que l'on trouve un 
encadrement britannique, mais il est vrai que nous avons souvent affaire ici à des compagnies londoniennes. L'exemple le plus emblématique reste la grande entreprise Dunderland Iron Ore Co. à Dunderlands Dalen, «sans doute la plus grande entreprise britannique en Norvège $»^{80}$, mais réellement active seulement de 1906 à $1908^{81}$, dont l'ingénieur électricien David A. Bremner (1866-1955) devient directeur-général. On le verra également en Suède, à la tête de la Hydraulic Power and Smelting Co. de Trollhättan ${ }^{82}$.

\section{Des expériences et des regards individuels}

Toute cette énumération de noms, qui nous a semblé nécessaire pour démontrer l'action de tous ces personnels britanniques, ne doit toutefois pas nous faire oublier que tous ces techniciens sont d'abord des êtres humains, doués de raisonnement, d'intérêts et d'émotions, dont l'expérience nordique, durable ou non, se construit à l'intérieur d'un parcours professionnel déjà entamé. Tout cela n'est pas sans effet sur le regard que ceux-ci portent sur ce qui est fréquemment vu comme une périphérie septentrionale.

Provisoire ou non, le séjour dans les régions nordiques est donc souvent dû aux hasards d'un cheminement professionnel. Pour la période qui nous intéresse, nous sommes loin du système organisé de « débauchage » de techniciens spécialisés anglais mis en place par certaines entreprises textiles norvégiennes au milieu du XIX ${ }^{\mathrm{e}}$ siècle et largement décrit par K. Bruland ${ }^{83}$. Compte tenu des nouveaux champs d'intervention, qui exigent un savoir-faire industriel bien plus complexe, nombre de ces ingénieurs sont envoyés par leur entreprise par suite de l'obtention d'un contrat ou d'une concession. Outre W.J. Walker Heath, déjà mentionné en Suède, c'est la Fox, Henderson \& Co., très active à l'Ouest de la Baltique, qui envoie William George Brounger (1820-1902) au Danemark dans les années 1850 pour prendre en charge la construction de la ligne ferroviaire entre Roskilde et Korsør ${ }^{84}$. De même, c'est l'entreprise Brassey \& Betts qui, dans les années 1860, envoie dans le même pays un ingénieur civil, Henry Hakewill (1842-1870), spécialisé dans la construction de ponts, dont le Westminster Bridge. Il y restera plusieurs années, employé à l'aménagement d'une ligne ferroviaire entre Horsens et Vejle, puis sur la ligne Aalborg-Randers ${ }^{85}$. Parfois même, il arrive qu'un ingénieur sollicite ou accepte un contrat dans le Nord, qui, à cette époque, représente une terre d'opportunités professionnelles. C'est le cas de William Lawford (1820-1903), déjà responsable de la construction de lignes en Grande-Bretagne. Celui-ci accepte en 1857 un poste sur la ligne Riga-Dünaburg, «sa connaissance de la langue allemande le désignant pour résider dans le pays » où il reste jusqu'en $1859^{86}$.

$\mathrm{Au}$ moins pour ce qui concerne ces «migrants provisoires ", il convient de mentionner que, du fait de la nature même de leurs missions, l'Europe du Nord n'est qu'une étape parmi d'autres dans le cadre d'un véritable parcours international pour ces ingénieurs, dont l'expertise technique est très recherchée. Dans le sillon des grandes célébrités de l'ingénierie civile britannique que sont S. Morton Peto, T. Brassey et J. Hawkshaw, nous avons donc affaire à une foule d'« ingénieurs itinérants ", en quelque sorte, que l'on retrouve partout dans le monde associés aux grands projets de travaux publics et de modernisation des infrastructures, en particulier dans les territoires britanniques et sur le continent américain. 
29 Néanmoins, le terme " migrant » prend tout son sens lorsque certains de ces hommes décident de rester plus longtemps sur place, voire de ne plus rentrer au pays, même si la rareté des sources personnelles ne nous permet pas de savoir ce qui les pousse à se fixer. Nous pouvons tout de même nous arrêter sur deux exemples :

30 A. Woodlands Makinson, déjà cité, a comme la plupart des personnes mentionnées ici, commencé sa carrière en Grande-Bretagne. Il a notamment travaillé sur le système de ventilation du parlement à Westminster. Il obtient également des contrats dans d'autres territoires britanniques et à l'étranger (en Suisse (1854), à Calcutta (1859), ou à Panama). Il arrive en Suède en 1871, où il mène à bien divers grands travaux ferroviaires, à la suite de quoi il décide de devenir fermier. Il meurt à Herrestad en $1886^{87}$

31 Samuel Betts (1841-1924), quant à lui, suivant encore le même schéma, commence sa carrière au début des années 1860 comme ingénieur en charge de la construction des locomotives aux usines Canada Works à Birkenhead (Liverpool), à destination de la ligne Dünaburg-Vitebsk. C'est peut-être la raison pour laquelle on le voit en Russie à partir de 1866, où il établit un atelier de réparation du matériel roulant à Vitebsk. On le retrouve par la suite responsable des locomotives de la ligne locale Riga-Bolderäja en 1870-1871, et au même poste en Suède (1873) pendant la construction de la ligne Oxelösund-Flen-Västmanland, puis jusqu'en 1910, date à laquelle il prend sa retraite, mais reste dans ce pays. Il meurt à Danderyd en $1924^{88}$.

32 La forte implication des entreprises britanniques citées ci-dessus est souvent liée non seulement aux bénéfices que l'on peut tirer de la construction d'une ligne ferroviaire, par exemple, mais aussi à ceux que l'on peut obtenir de son exploitation. Ceci nous intéresse ici en premier lieu lorsque certains de nos ingénieurs se trouvent également occuper un poste de dirigeant au sein de ces entreprises. En Norvège, c'est en partie ce qui pousse S. Morton Peto à engager son entreprise dans la construction de la ligne Christiania-Eidsvoll, en lien direct avec les régions forestières du lac Mjøsa ${ }^{89}$. Mais chez ce dernier les motivations purement personnelles prédominent parfois de manière flagrante. Son implication dans la Royal Danish Railway prévoyait en effet dès l'origine, en étendant le réseau, de relier tout le Danemark au port de Tönning, sur la mer du Nord, point de chute de sa propre ligne de navigation (North of Europe Steam Navigation Company); ceci en particulier dans le but d'exploiter en exclusivité le transport de bétail ${ }^{90}$.

Outre les motivations et les parcours propres à chaque individu, les rares sources auxquelles nous avons pu avoir accès quant aux sentiments personnels de nos protagonistes font parfois apparaitre un regard britannique à travers lequel se révèle également un jugement sur la main-d'œuvre locale moins monolithique et moins prisonnier des idées préconçues victoriennes et impérialistes que nous pourrions le croire au vu des articles de l'Engineer et des stéréotypes de l'époque ${ }^{91}$. Il est vrai qu'Harry Pasley Higginson (1838-1900), ingénieur sidérurgiste sur la ligne RigaDünaburg (1859-1861), montre dans ses mémoires les préjugés britanniques habituels sur les Russes, se complaisant dans la description de châtiments barbares dans un pays où «il $\mathrm{y}$ a très peu de justice $»^{92}$. Pourtant, cela n'empêche pas John Hawkshaw, son propre employeur, de reconnaître que «les ouvriers russes [...] apprennent vite et adoptent facilement toutes les méthodes dont on leur montre qu'elles sont meilleures que les leurs $»^{93}$. Ce n'est pas si mal pour un peuple réputé alors pour être obtus et lent d'esprit, théorie du climat oblige ! 

Baltique, où ce que disent T. Brassey et l'un de ses agents, Mr. Rowan, des ouvriers danois présents sur les chantiers de la Royal Danish Railway peut paraître surprenant. Qu'on en juge: "[Bien qu'] aucun homme ne puisse rivaliser avec un ouvrier britannique, [...] le Danois, par son travail régulier et constant, est un bon ouvrier; et un Danois de première classe [sic] accomplit en un jour presqu'autant de travail qu'un Anglais ». Selon Mr. Rowan, les Danois "prennent leur temps" pour exécuter leur travail (qu'ils font bien), ce qui fait écho à la lenteur, principale caractéristique que les Anglais attribuent volontiers aux Scandinaves en général, en plus d'un manque chronique d'esprit d'initiative dû, semble-t-il, à une trop grande éducation technique ${ }^{94}$, par opposition à l'ingénieur britannique type tel qu'il se voit : peu éduqué, mais bien plus proactif pour avoir appris sur le tas ${ }^{95}$.

On le voit, ce regard britannique, même s'il contient fatalement les stéréotypes contemporains tout en étant empreint d'un léger sentiment de supériorité ethnique, reste dans l'ensemble plutôt bienveillant. Ceci semble d'ailleurs confirmé par William Lloyd, envoyé en Suède en $1853^{96}$, pour qui les ingénieurs militaires suédois chargés de le seconder "se sont avérés non seulement agréables, mais parfaitement dignes de confiance et compétents $»^{97}$.

\section{De la migration à l'immigration}

La plupart de ces professionnels étaient venus pour une mission précise d'un temps limité. Ils s'en sont acquittés, puis s'en sont allés. Pourtant, il n'est pas interdit de se demander dans quelle mesure leur contact avec le Nord a pu influer sur l'existence de ces personnalités aux motivations et aux affects divers.

Comme le confirme Claus Wohlert dans le cadre de la métallurgie suédoise, le séjour des personnels industriels britanniques est la plupart du temps provisoire, au moment des étapes de construction en particulier, même s'il peut arriver que certains d'entre eux restent sur place parfois dix ou quinze ans ${ }^{98}$. Cette dimension transitoire est même constatée par les contemporains, comme le fait en 1859 le consul James Grignon à Riga $^{99}$; et le fait est que parmi tous les noms que nous avons utilisés pour constituer les Figures 1 et 2, dans l'immense majorité des cas cette analyse se trouve complètement corroborée. Nous avons mentionné certaines durées de séjours un peu longues concernant les ingénieurs évoqués dans cet article, mais d'autres personnes ne restent en effet que le temps de l'installation des machines ou de la construction des usines. Cela étant, si la principale explication de la réduction des effectifs britanniques en Scandinavie réside effectivement dans un retour au pays du personnel industriel, certains noms disparaissent des registres consulaires ou paroissiaux en ce sens que l'on assiste à une forme d'évaporation des communautés britanniques en tant que groupes homogènes en faveur d'une assimilation au sein de la communauté d'accueil, en particulier par le biais de mariages interethniques.

\section{Les mariages interethniques en tant que facteurs d'intégration}

Sur un plan plus général, Martin Åberg a conduit une étude intéressante sur les mariages interethniques (entre Britanniques et autochtones) dans la communauté britannique de Göteborg dans la deuxième moitié du XIX ${ }^{e}$ siècle en tant que facteurs et 
révélateurs d'intégration au sein de la population locale. Ses résultats, basés sur les registres paroissiaux de la British Factoryde la ville, révèlent que trente mariages sur trente-huit ont été des mariages interethniques entre 1850 et $1896^{100}$. Le même type d'étude sur les communautés britanniques à Saint-Pétersbourg et à Riga, à travers les quelques registres que nous avons pu consulter ${ }^{101}$, donne vingt-sept mariages interethniques sur quatre-vingt-quatre à Riga entre 1850 et 1919, et soixante et un sur deux cent soixante et un dans la capitale russe entre 1880 et 1914. Notons au passage que le nombre total de mariages enregistrés, interethniques ou non, a augmenté régulièrement sur toute la période, décennie après décennie.

Ainsi, même dans les régions éloignées de la Baltique orientale, les différences culturelles paraissent pouvoir être surmontées pour conduire vers une intégration relativement facile au sein de la population locale; phénomène déjà ancien comme en témoigne le nombre impressionnant d'Écossais, plus ou moins célèbres, déjà assimilés ${ }^{102}$. Cependant, il convient de garder à l'esprit que les statistiques que nous venons de présenter sur les mariages concernent les communautés dans leur ensemble, tous corps de métiers confondus, et donner le même type de proportions uniquement pour les effectifs industriels n'aurait que peu de sens, vu la proportion moindre de personnels qui restent sur place. Contrairement aux marchands ou aux entrepreneurs de type Baird, ceux-ci sont en effet rarement poussés à rester par une réussite économique locale à conserver. Il se trouve pourtant certains d'entre eux qui franchissent le pas :

Un certain William Graham, conducteur de locomotive, présent en Norvège entre 1854-1873, épouse en secondes noces une Norvégienne nommée Tonette Andersen. William Paisley, ingénieur civil, est, à la date de 1857, marié avec une certaine Inger Marie Aslaksen. Il en va de même pour James Mollat Smith, responsable du district ferroviaire de Christiania en 1869, époux d'une certaine Clara Marie Christophersen. Sans vouloir être exhaustif, d'autres exemples suivent, dont nous donnons les noms, le métier et les dates de présence attestée en Norvège: Henry Marmont, tisserand (1870-1879), avec A. Gustava Larsen, et Andrew Scotie, tisserand (1871-1879), avec Josephine Arnesen. Enfin, Robert Sinclair, ingénieur ferroviaire en 1878, avec Wilhelmina Larsen ${ }^{103}$.

Du côté russe, nous trouvons Benjamin Hall, ingénieur civil à Reval de 1876 à 1879, qui épouse une Russe en secondes noces: Olga Amalia C. Yvagona ${ }^{104}$. John Shaw, ouvrier aciériste à Libau en 1901, est, quant à lui, marié à Constanze Behr ${ }^{105}$. Nous avons également Benjamin Rayner, papetier à Riga en 1909, marié à une certaine Anna Vasilievna Friedlein ${ }^{106}$. Nous pouvons observer au passage que le mariage interethnique peut évidemment se faire avec les différentes souches présentes localement, germanique ou russe. Ce choix peut dépendre de la qualité homogamique de ces unions, en ce sens que, parmi les classes les plus élevées des colonies britanniques, le choix du conjoint se porte souvent sur des membres de la vieille aristocratie allemande, notamment dans les régions baltes. C'est ainsi le cas, assez connu, de George Armitstead (1847-1912), né, lui, au sein d'une des grandes dynasties britanniques de Riga, ingénieur, entrepreneur et surtout maire de la ville dans la première décennie du $\mathrm{XX}^{\mathrm{e}}$ siècle. Il épouse Cäcilie Anna Pychlau, née elle aussi à Riga, de la même famille allemande que sa propre mère ${ }^{107}$. Cet exemple d'alliance en circuit presque fermé entre deux grandes familles britanniques et allemandes, sans être majoritaire, n'est pas rare. 
Nous ne pouvons évidemment pas donner aux quelques cas évoqués ici une valeur axiomatique. Notons toutefois qu'outre le fait que ces mariages interethniques ne sont visiblement pas rejetés d'après les proportions que nous avons données, il est possible d'avoir une idée de la perception contemporaine à travers le constat affligé de l'aumônier de la Saint Saviour's Church (Riga) en 1914, qui nous apprend que «la colonie britannique est réduite [car] nombre de ses membres ont choisi leur conjoint parmi la population locale, et eux-mêmes et leur famille ont perdu tout contact avec les Anglais. $»^{108}$

\section{Conclusion} certains cas, ces hommes aux valeurs victoriennes profondément enracinées, possédant une forte conscience de soi pouvant aller jusqu'au complexe de supériorité, ces hommes, souvent venus à l'origine accomplir une mission d'ordre technique, arrivent à s'intégrer à travers des contacts socioculturels et économiques prolongés et à accepter, au moins pour partie, les codes culturels de la société d'accueil, surtout lorsque ceux-ci restent proches des codes culturels plus généraux des élites européennes. 


\section{NOTES}

1. Christiania est l'ancien nom d'Oslo.

2. House of Commons Parliamentary Papers (HCPP), Reports relative to British consular establishments, 1858 and 1871. Part II. 1872 [C.501], LX.317, p. 104, 111, 223 et 243. (422, 429, 541 et 561).

3. Philippe Blanc, La Grande-Bretagne, le Nord et la Baltique du milieu du XIX siècle jusqu'à la Première Guerre mondiale: des relations et un regard en évolution, thèse de doctorat en histoire contemporaine, université de Strasbourg, 2016, 1 vol. (699 p.), p. 166-167.

4. A.G Kenwood \& A.L. Lougheed, Technological Diffusion and Industrialization before 1914, Londres, Croom Helm, et New York, St Martin's Press, 1982, p. 132, 137, 141 et 156; David S. Landes, L'Europe technicienne ou le Prométhée libéré. Révolution technique et libre essor industriel en Europe occidentale de 1750 à nos jours, Paris, Gallimard, 2000 [1975], p. 208.

5. Robert Angus Buchanan, The Engineers. A History of the Engineering Profession in Britain 1750-1014, Londres, Jessica Kingsley Publishers, 1989, p. 147.

6. Eric Robinson, "The Transference of British Technology to Russia, 1760-1820", in Great Britain and Her World 1750-1870, Barrie Ratcliffe (dir.), Manchester, Manchester University Press, 1975, p. 7-10, 13 et 15 .

7. Ibid., p. 12-13 ; T. Tower, Memoir of the Late Charles Baird, Esq., of St Petersburgh, and of his Son, the Late Francis Baird, Esq., Londres, Harrison \& Sons, 1867, p. 2-6.

8. Pour un panorama plus complet, lire Emil Sahlin, British Contributions to Sweden's Industrial Development : some Historical Notes, Stockholm, The General Export Association of Sweden, 1964, $121 \mathrm{p}$.

9. Jonas Berg et Bo Lagercrantz, Scots in Sweden, Stockholm, Nordiska Museet \& The Swedish Institute, 1962, p. 54.

10. Emil Sahlin, op. cit., p. 59-60 ; Martin Fritz, "British Influence on the Developments in the Swedish Foundry Industry around the Turn of the Eighteenth Century", in Technology Transfer and Scandinavian Industrialisation, Kristine Bruland (dir.), New York, Oxford, Berg, 1991, p. 67.

11. Arne Sundström, "Samuel Owen, teknik- och ångbåtspionjär”, Daedalus, Stockholm, Tekniska Museets Årsbok, 2009, p 53-68 ; Emil Sahlin, ibid., p. 23 et 56-57. Voir également Eli Heckscher, An Economic History of Sweden, Cambridge (Mass.), Harvard University Press, 1954, p. 248.

12. Karl A. Lundkvist, "Den malkolmska verkstaden i Norrköping 1836-1868", Daedalus, Stockholm, Tekniska Museets Årsbok, 1976, p. 35 ; Emil Sahlin, ibid., p. 74-75 et 112.

13. William O. Henderson, Britain and Industrial Europe 1750-1870, Studies in British Influence on the Industrial Revolution in Western Europe, Leicester, Leicester University Press, 1965, p. 5.

14. Lars Magnusson, An Economic History of Sweden, Londres, New York, Routledge, 2000, p. 53-54 et $144-147$.

15. Formule empruntée à Ronald Robinson et John Gallagher, "The Imperialism of Free trade", Economic History Review, ${ }^{\circ}$ 6, 1, 1953, p. 1-15.

16. L'article fait explicitement référence aux travaux de John Hawkshaw en Russie baltique et de Charles Blacker Vignoles en Ukraine (n.d.a.).

17. “A New Russian Field of Enterprise”, The Engineer, 25 avril 1856, p. 229.

18. T. Tower, op. cit., p. 16-17.

19. Robert Angus Buchanan, op. cit., p. 157.

20. Sources : MPICE ; MPIME ; RG 33/153 ; LMA, MS 22402 ; FO 529/8 et 9 ; Emil Sahlin, op. cit.; Fritz Hodne, "Transfer Patterns of Technology", in Kristine Bruland (dir.), 1991, p. 167-168 ; Kristine Bruland, "The Norwegian Mechanical Engineering Industry and the Transfer of Technology", in Kristine 
Bruland (dir.), 1991, op. cit., p. 259-260. Selon les dates indiquées par nos sources, certaines personnes peuvent être présentes sur plusieurs décennies (n.d.a.).

21. Sources : FO 99/12, Register of Births. British Vice-Consulate at Pernau (Pärnu) ; FO 661/4, Register of Births, Libau; FO 769/1, Register of Births. British Vice-Consulate at Tammerfors; FO 377/3, 377/4, 378/4, 378/8, 514/7 et 514/8 ; LMA, MS 10953C/002 ; John R. Bowles, "From the Banks of the Neva to the Shores of Lake Baikal: Some Enterprising Scots in Russia", in The Caledonian Phalanx, Scots in Russia, E.F.D. Roberts (dir.), Édimbourg, National Library of Scotland, 1987, p. 70-75 ; Alan S. Milward \& S.B. Saul, The Development of the Economies of Continental Europe 1850-1914, Londres, George Allen \& Unwin, 1977, p. 494; Dimitry Fedosov, The Caledonian Connection, Scotland-Russia Ties, Aberdeen, Centre for Scottish Studies, University of Aberdeen, 1996. Le terme «Russie baltique » recouvre essentiellement le Grand-Duché de Finlande et les régions de Saint-Pétersbourg, Riga, Narva, Libau et Reval (n.d.a.).

22. Archives nationales britanniques: FO (Foreign Office) 83/111, folio 16, rapport du consul J.R. Crowe sur les maisons commerciales établies en Norvège en 1848.

23. Karl A. Lundkvist, op. cit., p. 35.

24. Lars Magnusson, op.cit., p.129-130; Kristine Bruland, British Technology and European Industrialization: The Norwegian Textile Industry in the Mid Nineteenth Century, Cambridge, Cambridge University Press, 1989, ix-193 p.

25. Martin Fritz, op. cit., p. 66.

26. Karl A. Lundkvist, op. cit., p. 35-36; Minutes of the Proceedings of the Institution of Civil Engineers (MPICE), vol. 115, 1894, p. 392, et vol. 150, 1902, p. 429; Minutes of the Proceedings of the Institution of Mechanical Engineers (MPIME), année 1902, p. 379.

27. Claus Wohlert, "The Introduction of the Bessemer Process in Sweden", in Kristine Bruland (dir.), 1991, op. cit., p. 301.

28. Kristine Bruland, 1989, op. cit., p 65.

29. Lars Magnusson, op. cit., p. 54.

30. William O. Henderson, op. cit., p. 212.

31. Sydney Pollard, Peaceful Conquest. The Industrialization of Europe 1760-1970, Oxford, Oxford University Press, 1981, p. 158 ; A.G. Kenwood \& A.L. Lougheed, op. cit., p. 151.

32. Stuart Thompstone, "The Russian Technical Society and British Textile Machinery Import", Nottingham, University of Nottingham, 2012, p. 3-5 et 8 (article PDF).

33. Ibid., p. 2, 9 et 12 ; Alan S. Milward \& S.B. Saul, op. cit., p. 356.

34. Kristine Bruland, "Skills, Learning and the International Diffusion of Technology: a Perspective on Scandinavian Industrialization", in Technological Revolutions in Europe, Historical Perspectives, Maxime Berg \& Kristine Bruland (dir.), Cheltenham, Edward Elgar, 1998, p. 180.

35. HCPP, Reports relative to British consular establishments, 1858 and 1871. Part II. 1872 [C.501], LX. 317, p. 117 (435) ; et Leland Hamilton Jenks, The Migration of British Capital to 1875, Londres, Édimbourg, Paris, Melbourne, Johannesbourg, Toronto et New York, Thomas Nelson and Sons Ltd, 1963 [1927], p. 184.

36. Stuart Thompstone, op. cit., p. 6.

37. Natalia Gurushina, "Free-Standing Companies in Tsarist Russia", in The Free-Standing Company in the World Economy 1830-1996, Mira Wilkins \& Harm Schröter (dir.), Oxford, Oxford University Press, 1998, p. 174.

38. Leland Hamilton Jenks, op. cit., p. 183 ; Martin Varley, "The Thornton Woollen Mill, St Petersburg", History Today, $n^{\circ}$ 44, 12, 1994, p. 62.

39. Stuart Thompstone, op. cit., p. 23-24.

40. Lars Magnusson, op. cit., p. 114.

41. Malcolm Falkus, "Aspects of Foreign Investment in Tsarist Russia", Journal of European Economic History, ${ }^{\circ}$ 8, 1, 1979, p. 8 et 12. 
42. John C. Jeaffreson, The Life of Robert Stephenson, FRS, Londres, Longman, Roberts \& Green, 1864, vol. I, p. 239, et vol. II, p. 130-131.

43. John G. Cox, Samuel Morton Peto (1809-1889), the Achievements and Failings of a Great Railway Developer, Oxford, Railway \& Canal Historical Society, 2008, p. 75 ; Sima Lieberman, The Industrialization of Norway 1800-1920, Oslo, Bergen, Tromsø, Universitetsforlaget, 1970, p. 95.

44. MPICE, vol.57, 1879, p. 300 ; Arthur Helps, Life and Labours af Thomas Brassey 1805-1870, Londres, George Bell \& Sons, 1888, p. 87-89 ; John G. Cox, ibid., p. 77.

45. MPICE, vol. 99, 1890, p. 400-403.

46. MPICE, vol. 36, 1873, p. 285-287.

47. John G. Cox, op. cit., p. 76.

48. Ibid., p. 77 ; Robert Keith Middlemas, The Master Builders, Thomas Brassey, Sir John Aird, Lord Cowdray, Sir John Norton-Griffiths, Londres, Hutchinson, 1963, p. 74.

49. MPICE, vol. 106, 1891, p. 321-335; et Martin Beaumont, Sir John Hawkshaw 1811-1891, the Life and Work of an Eminent Victorian Engineer, Lancashire \& Yorkshire Railway Society, 2015, p. 72.

50. Emil Sahlin, op. cit., p. 60.

51. The Engineer, 27 mars 1908, p. 318.

52. Dimitry Fedosov, op. cit., p. 78, 80 et 114.

53. Juhan Kahk \& Enn Tarvel, An Economic History of the Baltic Countries, Stockholm, Almqvist \& Wiksell International, 1997, p. 98.

54. HCPP, Reports from HM Diplomatic and Consular Offices Abroad on Trade and Finance (Full Version), 1890-91 [C.6205], LXXXV.1, LXXXVI.1, LXXXVII.1, LXXXVIII.1, n 860. Russia. Report for the Year 1890 on the trade of the Consular District of Riga, p. 15.

55. HCPP, Commercial Reports Received at the Foreign Office from Her Majesty's Consuls in 1867, 1867 [3762], LXVII.301, p. 7 (309).

56. Emil Sahlin, op.cit., p. 86-87 ; Arne Kaijser, "City Lights: the Establishment of the First Swedish Gasworks", Flux, n 6, 1, 1990, p. 80-81.

57. Archives nationales britanniques : RG (Records of the General Register Office) 33/153, $n^{\circ} 14$.

58. MPICE, vol. 97, 1889, p. 400-401.

59. MPICE, vol. 117, 1894, p. 369-374.

60. MPICE, vol. 168, 1907, p. 353-354.

61. MPIME, vol. 154, 1946, p. 133.

62. MPICE, vol. 198, 1914, p. 350-351; Martin Beaumont, op. cit., p. 72.

63. Martin Beaumont, Ibid.

64. MPICE, vol. 103, 1891, p. 380.

65. MPICE, vol. 39, 1875, p. 276-277 ; Emil Sahlin, op. cit., p. 42-43.

66. MPICE, vol. 30, 1870, p. 472.

67. Archives nationales britanniques: BT (Board of Trade) 31/14421/5192, The Swedish Central Railway Company, Limited ; Emil Sahlin, op. cit., p. 44.

68. MPICE, vol. 86, 1886, p. 356.

69. MPICE, vol. 117, 1894, p. 392-393; BT 31/3196/18591/12, The Northern of Europe Railway Company, Limited; Emil Sahlin, op. cit., p. 39.

70. MPICE, vol. 92, 1888, p. 404.

71. FO 378/4, Register of Births, British Consulate at St. Petersburg (Petrograd) (1856-1917).

72. Ibid.

73. FO 378/8, Register of Deaths, British Consulate at St. Petersburg (Petrograd) (1905-1917).

74. Ibid.; Martin Varley, op. cit., p. 62-63.

75. FO 514/8, Consular Register of Deaths, District of Reval (1875-1912).

76. FO 377/3, Register of Births, British Consulate at Riga.

77. Fritz Hodne, op. cit., p. 167.

78. The Engineer, 30 juillet, 1954, p. 164, et 19 février 1875, p. 132. 
79. MPICE, vol. 166, 1906, p. 384-385.

80. FO 368/315/39835, General Report on Norway for 1906, p. 16.

81. HCPP, Iron ore deposits in foreign countries. Reports on iron ore deposits in foreign countries compiled at the Board of Trade from information collected by H.M. diplomatic consular officers. 1905 [Cd. 2553], LXXXV.205, p. 130 (334); Michael Flinn, "Scandinavian Iron Ore Mining and the British Steel Industry 1870-1914", Scandinavian Economic History Review, n² 2, 1, 1954, p. $42-44$; Sima Lieberman, op. cit., p. 125.

82. MPIME, année 1955, p. 484.

83. Kristine Bruland, 1989, op. cit., p. 65 et 90-93.

84. MPICE, vol. 112, 1893, p. 359, et vol. 147, 1902, p. 412.

85. MPICE, vol. 31, 1871, p. 245-246.

86. MPICE, vol. 154, 1903, p. 377.

87. MPICE, vol. 86, 1886, p. 356.

88. MPIME, 1924, p. 703-704.

89. John G. Cox, op. cit., p. 75.

90. Ibid., p. 78-79; Robert Keith Middlemas, op. cit., p. 74.

91. Philippe Blanc, op. cit., p. 55-60.

92. Dossier of Papers Compiled to Mark the Occasion of the Centenary of the Death of Harry Pasley Higginson (archives de l'Institution of Civil Engineers) ; MPICE, vol. 142, 1900, p. 357.

93. Arthur Helps, op. cit., p. 51.

94. Ibid., p. 122-124.

95. Ben Marsden \& Crosbie Smith, Engineering Empires. A cultural History of Technology in NineteenthCentury Britain, Basingstoke, Palgrave Macmillan, 2005, p. 235-237.

96. Michael Bailey, "Wider Horizons", in Robert Stephenson, the Eminent Engineer, Michael Bailey (dir.), Londres, New York, Routledge, 2003, p. 148.

97. William Lloyd, Railway Engineers Experiences, vol. 1, p. 84, 85 et 93 (manuscrit déposé aux archives de l'Institution of Civil Engineers).

98. Claus Wohlert, "The Introduction of the Bessemer Process in Sweden", in Kristine Bruland (dir.), 1991, op. cit., p. 303.

99. FO 65/587/22, mémorandum du consul James Grignon (Riga) à Lord Russell du 26 septembre 1861.

100. Church records, $\mathrm{C}: 1-\mathrm{C}: 2, \mathrm{E}: 1$. The British Factory, Landsarkivet i Göteborg, cité par Martin Åberg, "Migration, Civic Culture and Politics: British Middle-Class Communities in Western Sweden 1730-1900", in Britain and the Baltic, Studies in Commercial, Political and Cultural Relations 1500 - 2000, Patrick Salmon et Tony Barrow (dir.), Sunderland, University of Sunderland, 2003, p. 183.

101. FO 378/4 ; FO 378/8; RG 33/146, registres de la British and American Church de SaintPétersbourg (1858-1885) (baptêmes, mariages et décès) ; FO 377/3 ; et FO 377/4, Register of deaths, British Consulate at Riga (1850-1912).

102. Voir Dimitry Fedosov, op. cit., p. 122.

103. FO 529/8 et 529/9, Register of births. British at Christiania (1849-1869 et 1869-1916).

104. FO 514/7, Consular Register of Births, District of Reval (1876-1911).

105. London Metropolitan Archives (LMA) : MS 10953C/002 : Register of marriages, Libau (Courland).

106. FO $378 / 4$

107. FO 377/3 ; LMA, MS 11228 ; Gert von Pistohlkors, "Germany and the Baltic Region in the Short Twentieth Century", in Germany and the European East in the Twentieth Century, Eduard Mühle (dir.), New York, Berg, 2003, p. 46.

108. LMA, MS 11228, Records of Saint Saviour's Church, Riga, p. 57.

109. Philippe Blanc, op. cit., p. 99 et 232-242.

110. FO $377 / 3$. 


\section{RÉSUMÉS}

Le milieu du XIX ${ }^{\mathrm{e}}$ siècle voit à la fois la libéralisation totale de l'exportation de technologie britannique et une Europe du Nord en pleine phase d'industrialisation, véritable champ de conquête pour l'ingénierie britannique, alors à son apogée. Nous nous proposons d'examiner, dans ce contexte, quels ont été les domaines et les évolutions de la contribution des industriels et ingénieurs britanniques dans cette région, aux conditions socioéconomiques locales très différentes, durant toute la deuxième moitié de ce siècle jusqu'à la Première Guerre mondiale. Nous nous focaliserons également sur certains intervenants eux-mêmes, non seulement en tant que représentants d'une grande puissance industrielle, mais aussi parce que c'est avec leurs propres préjugés et leur propre parcours que ces hommes ont participé à un mouvement migratoire sur la durée de plusieurs centaines d'individus; ce qui entraîne la question de savoir si cette aide humaine à l'industrialisation a pu parfois conduire à une véritable intégration au sein des sociétés d'accueil.

The middle of the $19^{\text {th }}$ century saw the total liberalization of exports in British technology while Northern Europe was becoming industrialized and a field of conquest for British engineering, then in its heyday. We would like to examine, in this context what were the various domains and developments of the contribution of British industrialists and engineers in this region, in very different socioeconomic local environments, throughout the second half of the century until the First World War. We will also focus on some of the engineers themselves, not only as representatives of a major industrial power, but also because they were men who, having their own prejudices and careers, took part in a continuous migratory movement of several hundred individuals; which raises the question of whether this human assistance to industrialization may at times have led to actual integration within the host societies.

\section{INDEX}

Mots-clés : mouvement migratoire, industrialisation, Europe du Nord, Grande Bretagne Keywords : migratory movement, industrialization, Northern Europe, Great Britain

\section{AUTEUR}

\section{PHILIPPE BLANC}

Philippe Blanc est PRAG d'anglais à l'université de Bourgogne-Franche-Comté. Il est docteur en Histoire contemporaine - UMR 7367, Dynamiques Européennes (Strasbourg). 